\title{
Innovation in practice: Mobile phone technology in patient care Holly Blake
}

Faculty of Medicine \& Health Sciences, University of Nottingham, UK.

Citation: Blake H: Innovation in practice: mobile phone technology in patient care. Br J Community Nurs; 2008 Apr;13(4):160, 162-5

DOI: http://dx.doi.org/10.12968/bjcn.2008.13.4.29024

Published Online: September 27, 2013

Contact: Holly.Blake@nottingham.ac.uk

Key words: Technology, mobile phone, monitoring, health communication.

\begin{abstract}
Mobile phones are becoming increasingly important in everyday life and now in healthcare. There has been a steady growth of information and communication technologies in health communication and technology is used progressively in telemedicine, wireless monitoring of health outcomes in disease and in the delivery of health interventions. Mobile phones are becoming an important method of encouraging better nurse-patient communication and will undoubtedly increase in application over coming years. This article presents recent developments and applications of mobile technology for health promotion and patient monitoring in chronic disease.
\end{abstract}


Whether aiming to improve health at the individual, community, regional or national level, increasing developments in the new millennium have seen a steady growth of information and communication technologies in virtually all areas of health communication. Technologies are used in research and education, knowledge transfer, social support, service delivery and health promotion. Mobile technology is increasingly used in telemedicine, wireless monitoring of health outcomes in disease management and delivery of health interventions. Mobile phones are emerging as an important method of encouraging better nurse-patient communication and are estimated to increase in use and application over coming years.

\section{Health promotion}

In recent years, there has been an increase in the development of health promotion programmes which utilize the text message or voice response function of the mobile phone, either as an intervention per se, or in combination with other technologies. These have been conducted mostly in Japan, the US, New Zealand and more recently, the UK, and have included interventions for diet or weight management, physical activity, alcohol and drug use and smoking cessation, many of which have shown positive outcomes.

\section{Dietary intervention}

Several Japanese studies have reported the use of mobile phones for dietary programmes. In 2004, a health education programme named "i-exerM" was developed in collaboration with commerce to deliver a 12-week body weight reduction programme to 136 adults (Kubota et al, 2004). Educational items about body weight reduction were sent by text message to participants on a daily basis and they were asked to record their body weight periodically on an internet website. There was a tendency for reduced body weight in those who participated and the programme was well-evaluated.

More recently, Wang et al (2006) evaluated a new dietary assessment method which consisted of a hand-held personal digital assistant with camera and mobile telephone card, called Wellnavi. Previous work by this team had suggested in a small sample that the Wellnavi could usefully measure individual dietary intakes for a variety of nutrients (Wang et al, 2002). In a cross-sectional study in 2006, participants were asked to keep 1-day weighed food records. Digital images of all recorded foods were obtained simultaneously and sent to registered dietitians by a mobile telephone card. Estimated median nutrient intake was compared across methods and the Wellnavi compared well with food records for most nutrients with some exceptions. Over half of the participants reported that the Wellnavi was the least burdensome method of assessment and the least time-consuming. Half the sample reported that they would be willing to record their diet in this way for a month. The authors concluded from this work that this system may be a valid and convenient method for recording diet. Later work highlighted potential problems 
with this method. Kikunaga et al (2007) compared Wellnavi with a weighed diet record as a reference method. They also looked at the relation between obesity and underreporting when using the Wellnavi method in the general population. In this study however, the authors found that the values of daily nutrient intakes in the Wellnavi method were lower than those estimated by the weighed diet record method and this seemed to be due to the low quality of the digital photo of the Wellnavi instrument. It may be that this type of instrument may have potential for gaining dietary information from a diverse population and thus be of interest to healthcare professionals, although further work needs to be done to improve the digital photo quality of the Wellnavi.

\section{Smoking cessation intervention}

Wireless text messages have shown promise for the deliver of smoking cessation interventions. Obermayer et al (2004) developed and evaluated a prototype program targeting US college students that integrated Web and cell phone technologies to deliver a smoking-cessation intervention $(n=46$ regular smokers). This individualized quitting program was delivered by means of cell phone text messaging with assessment tools delivered using the program Web site. At sixweek follow-up, one fifth of the sample had quit smoking based on seven-day prevalence critieria and $43 \%$ had made at least one 24 -hour attempt to quit. Lazev et al (2004) looked at the feasibility of using mobile telephones to improve access to smoking cessation counseling in a low-income, HIV-positive population. The authors reported findings from two studies - the first assessed the interests and barriers towards such an intervention $(n=49)$. The second evaluated the impact of an intervention in which a small sample of participants $(n=20)$ were given free mobile telephones and received six telephone counseling sessions over a two-week period. Participants in the first study expressed interest in mobile phone intervention although identified phone access as a barrier. In the second study, nineteen participants completed the intervention and all of these made a quit attempt, with $75 \%$ point-prevalence abstinence rate at the end of the two weeks. This work showed potential for the provision of a proactive mobile telephone smoking cessation intervention which could provide an underserved population with access to care. Further work with this target group suggests that individuals living with HIV/AIDS are receptive to, and can be helped by, smoking cessation treatment (Vidrine et al, 2006a). In a randomised clinical trial $(n=95)$, this mobile phone based smoking cessation intervention was compared with usual care after the intervention and again at three month follow-up $(n=77)$. The usual care group received brief physician advice to quit smoking, targeted self-help written materials and nicotine replacement therapy. The cellular telephone intervention received the eight counseling sessions delivered via mobile telephone in addition to the usual care components. Participants receiving the mobile phone intervention were 3.6 times more likely to quit smoking than those in receipt of usual care and these results were biochemically verified. In a second article, Vidrine et al (2006b) reported that the mobile phone intervention group experienced greater reductions in anxiety and depression, and increases in self- efficacy compared with the usual care group. 
In New Zealand, a single-blind randomised controlled trial was conducted which targeted the recruitment of young Maori (Bramley et al, 2005). The research team recruited participants who were interested in giving up smoking within one month (aged over 16 years) and who had access to a mobile phone network. The intervention group received regular, personalised text messages providing smoking cessation advice, support, and distraction. Maori text messages related to Maori language, messages (in Maori and English) and information on Maori traditions. Text messaging was free for the intervention participants for one month. There were 1705 participants (355 Maori and 1350 non-Maori). At the six week follow-up, Maori receiving the intervention were more likely to have quit than Maori in the control group. The intervention was just as effective for Maori as for non-Maori and there were no significant differences between the two at any time point. This mobile phone smoking cessation programme demonstrated that a text messaging intervention can have a positive effect for short-term, self- reported quit rates. However, the findings are limited by an over-reporting of quit rates (possibly due to the timing of free text messaging incentives) and a differential loss to follow up, with increased attrition in the intervention group at 26 weeks.

A further New Zealand study examined the effectiveness of a mobile phone messaging service (Rodgers et al, 2005) in 1705 smokers who wanted to quit. The intervention group received regular, personalized text messages providing smoking cessation advice, support, and distraction. All participants received a free month of text messaging; starting for the intervention group on their quit day to assist with quitting, and starting for the control group at six months to encourage follow up. Follow up data were available for 1624 (95\%) at six weeks and 1265 (74\%) at six months. Smoking habits were assessed at six, 12 and 26 weeks. More participants had quit at six weeks in the intervention compared to the control group (28\% versus $13 \%$ ) and this treatment effect was consistent across subgroups defined by age, sex, income level, or geographic location. This difference was still evident at 12 weeks but not at 26 weeks. However, at 26 weeks, the smokers in the intervention group were more likely to smoke less cigarettes per day and be at a higher stage of preparedness to change than smokers in the control group. Of participants who reported quitting at 26 weeks, those in the intervention group were more confident about staying quit compared to those in the control group who reported quitting. This research showed that mobile phone intervention may be successful in smoking cessation although this needs to be tested further in different settings and with increased emphasis on achieving long-term quit rates.

\section{Alcohol and substance use intervention}

Collins et al (2003) looked at the feasibility of using mobile phones to collect ecological momentary assessment data for alcohol consumption. In this US study, social drinkers $(n=20)$ were randomly assigned to either paper-and-pencil selfmonitoring (PM) or cellular phone monitoring (CM) and their drinking was monitored for two weeks. The PM group recorded their drinking behaviours on cards and the CM group responded to an interactive voice response system. 
Although alcohol use, compliance and satisfaction did not differ between groups, there were several benefits to using the mobile phone system. These included the ease of integration into everyday life, instantaneous entry of data into a central database and accurate date/time records of data. This suggests that mobile phone monitoring for alcohol consumption may be a useful alternative to paper- based intervention.

Mobile phones have also proved useful in accessing previously hard to reach groups. For example, individuals without connected land-line telephones are often missed in computer-assisted telephone interview (CATI) household survey methodology. Wilkins et al (2003) adapted a telephone based survey for the mobile phone and found that individuals in households without connected land- line telephones drank significantly more alcohol even when consumption levels were controlled for socioeconomic status. Although there are high levels of telephone ownership in the general population, this work shows that mobile phones may be used to access specific subpopulations where land-line telephone ownership may be lower.

Ecological momentary assessment data (EMA) has also been collected in specific target groups. Freedman et al (2006) used mobile phones and computer- automated telephone interviewing to collect EMA with cocaine-addicted homeless patients in treatment $(n=30)$. Participants treated in an intensive outpatient treatment program were given mobile phones for a two-week period to record current states of cocaine craving and using episodes. Eighty per cent of the sample completed the two week protocol and this method of data collection proved reliable and acceptable to users. Drug use reported via EMA was in agreement with urine toxicology results for $73 \%$ of participants. This type of mobile phone intervention may therefore have potential with drug-addicted homeless persons in treatment.

\section{Physical activity intervention}

Several intervention programmes are currently being developed to increase physical activity in the general population. Consolvo et al (2006) report the development and testing of a mobile phone-based fitness journal, „Houston', to track and share progress toward a daily step count goal within a small group of friends $(\mathrm{n}=13)$. This system is based on the concept that health benefits can occur simply from increasing daily steps and social support can be a motivating factor in increasing physical activity. Despite study limitations this work did suggest that such an intervention may be experienced positively by users provided that it gives users credit for their activities, provides personal awareness for activity levels, supports social influence and considers the practical constraints of users lifestyles.

A recent study evaluated the use of internet and mobile phone technology to deliver a physical activity programme with 77 adults (Hurling et al, 2007). This study was a randomised controlled trial which compared a fully automated, nine week intervention $(n=47)$ with a no treatment control condition $(n=30)$. Participants in the intervention group received tailored solutions for perceived barriers, a schedule 
to plan weekly exercise sessions with mobile phone and email reminders, a message board to share their experiences with others, and real-time feedback on their level of physical activity via the Internet. Physical activity was measured in both groups using a wrist-worn accelerometer and self-report questionnaires. Self-reports and objective data showed that moderate level activity had increased in the intervention group. The intervention was successful and showed that a fully automated system incorporating mobile phones could both increase and maintain physical activity in adults.

\section{Health monitoring}

Patient symptoms, health status and quality of life are increasingly monitored in this way. Research studies have been conducted that test the feasibility of these methods with patient groups. These have been predominantly conducted in Europe (Italy, Denmark, Norway, Spain, UK), Korea and the USA. Interventions have mainly focused on diabetes management, with some studies on cancer, asthma and healthcare of older people.

\section{Cancer}

Bielli et al (2004) developed a Wireless Health Outcomes Monitoring System (WHOMS). This system created a method whereby structured questionnaires could be sent directly to the patient"s mobile phone by their medical management team. Patient"s answers are automatically transferred to an authorized website which then displays the patient"s current state of health in graphical form, accessible by the medical team. The WHOMS was tested with 97 cancer inpatients and whilst more than half completed the questionnaire successfully, the non- responders were often older, had fewer years of education and were less familiar with new communications technology (mobile phone calls, mobile phone SMS, internet, email). This evaluation work demonstrated that distance monitoring using mobile phones is plausible and health information can be collected from a large proportion of patients in this way. This proportion may increase in community settings if the family were involved to assist the patient with the technology. However, this type of technology may not be accessible to all patient groups.

\section{Asthma}

Such a system is likely to detect patient suffering earlier, and to activate a welltimed intervention. Internet technology is currently used for patient monitoring, however, web-based asthma diaries have been criticized for high rates of attrition. Mobile phones have been tested as an alternative to Web interface in the monitoring and self-management of asthma. Anhǿj and Mǿldrup (2004) used the short message service (SMS) for asthma diary data collection in which patients $(n=12)$ were sent four SMS messages per day and were encouraged to reply to at least three of them daily. Messages included a medication reminder, a request to enter peak flow, data on sleep loss and medication dosage. Responses were steady during the study 
period and did not decrease over time, with more than half of the participants reporting two-thirds of the requested data. This indicates that mobile phones may be a feasible method for self-monitoring although focus groups with participants suggest that daily messages requiring responses should reduce in number and also that graphical display of diary data would be preferred by patients. Mobile phones may therefore be a practical support for self-management of asthma although may be most effective in combination with web-based monitoring rather than as a replacement.

\section{Diabetes}

New communications systems have been used for the self-management of adult and child patients with diabetes who undergo rigorous procedures for blood glucose monitoring and regulation. In 2002, a Spanish study looked at Internet and mobile phone use in adults with Type 1 diabetes (Giménez-Pérez et al, 2002). The researchers interviewed 244 participants ( 115 men, 129 women) and found a low rate of access and utilization of the Internet for health-related purposes with only a third of the sample reporting that they regularly used the Internet for health-related purposes. However, of the $76.6 \%$ of the patients that owned a mobile phone, $96 \%$ used it more than once a week showing a high rate of ownership and use of mobile phones. Mobile phones therefore showed promise as tools in health communication and self-management of disease.

In the UK, Farmer et al (2005) describe the development and implementation of an innovative real-time telemedicine system based around transmission and feedback of data to and from a mobile phone. This intervention included the immediate transmission of blood glucose data from a blood glucose monitor and collection of information about physical activity, eating patterns and insulin dose. Participants received immediate feedback to their phone which included a colour histogram mapping levels of control over glycaemia over the previous two weeks. Clinicians supporting patients had access to summary screens which allowed them to identify users who were not testing, and those with levels of blood glucose which were outside pre-defined limits. There were more detailed graphical displays of data and these were used to provide data about control of insulin dose and the degree to which it was modified in response to diet and exercise. This system has been used with success in both primary and secondary care environments.

Recent work by Kim and Jeong (2007) looked at the effectiveness of a nurse short message service (SMS) by cellular phone and wire Internet on plasma glucose levels in people with Type 2 diabetes for a period of six months. In this controlled study, participants were randomly allocated to either the intervention $(n=25)$ or the control group $(n=26)$. With the aim of keeping blood glucose concentrations to the normal range, participants in the intervention group were requested to input their blood glucose level, diet and exercise diary daily in the website by mobile phone or wire Internet. The researcher sent optimal recommendations to each patient by mobile phone SMS text messaging, and wire Internet on a weekly basis. In this study, 
glycosylated hemoglobin $(\mathrm{HbA}(1) \mathrm{c})$ decreased $1.15 \%$ points at three months and $1.05 \%$ points at six months compared with baseline in the intervention group. Patients in the intervention group had a decrease of two hours post meal glucose (2HPMG) of $85.1 \mathrm{mg} / \mathrm{dl}$ at three months and $63.1 \mathrm{mg} / \mathrm{dl}$ at six months compared with baseline. This study showed that a nurse-led mobile phone intervention can reduce $\mathrm{HbA}(1) \mathrm{c}$ and $2 \mathrm{HPMG}$ for six months in type-2 diabetic patients and results were confirmed by Kim (2007).

Mobile telecommunication systems have been used in the care of children with Type 1 diabetes. In Norway, Gammon et al (2005) demonstrated how mobile phones could be used to increase interaction between children and their parents by developing a mobile and wireless system which automatically transferred readings from a child"s blood glucose monitor to their parent"s mobile phone. The researchers evaluated parent $(\mathrm{n}=30)$ and child $(\mathrm{n}=15)$ experiences of the system through questionnaire surveys and interviews. The children using the system were aged $9-15$ years. The authors found that the system was easily integrated into everyday life and was particularly effective when children measured their blood glucose levels regularly. In these cases it seemed that the system aided parents with attaining the balance between control of children"s monitoring procedures and allowing their independence. However, for those children who measured their blood glucose irregularly, parental reminders ("nagging") increased. Parents also felt that the system was less appropriate in adolescence where the system could generate a potential sense of "surveillance" which could lead to oppositional behaviour. On the whole however, the system was well- received and although it is not know if it impacted on actual health outcomes, the study suggests there may be a consumer market for these systems and parents in the study suggested the possibility for similar alerts of irregular insulin dosages and automatically generated dietary and insulin dosage advice.

A similar system used with childhood diabetes has been developed more recently in the USA. Carroll et al (2007) developed a prototype system, the HealthPia GlucoPack Diabetes Monitoring System, which integrates a small blood glucose monitoring device into the battery pack of a mobile phone. In a pilot study, the authors evaluated satisfaction with use of the system and also looked at the potential for the device to transmit self-monitoring data to a website for review and analysis by clinicians, parents, and patients. The adolescents in this study found the system easy to use and useful in the self-management of their diabetes. This group also reported liking the integration of technologies for this purpose.

Mobile technologies have also been applied in healthcare of older people. Miskelly (2005) reported the use of mobile phone alerts for the electronic tracking of patients with dementia and wandering and results were promising. However, there is currently little published research in this area and more evidence is needed for the application of mobile technology in this way. 
Conclusion

A large proportion of the UK now owns or has access to a mobile phone. Many mobile phone applications have the potential to make a major contribution to health promotion and patient care through the communication of health messages aimed at the modification of lifestyle behaviours, and also in the monitoring of patient health data. This may assist with population interventions to improve health or in chronic disease management to assist with the timeliness of medical intervention. Mobile data collection may include self-report questionnaire data on health and lifestyle behaviours, or objective measures such as blood pressure, cardiac pulsation, ECG, weight, step counts and so on. These may be delivered as specific mobile phone interventions, or multicomponent interventions incorporating other technologies such as email and Internet. Mobile phones are encouraging a more dynamic connection between healthcare staff, patients and child-parent dyads. They have the potential to be cost-effective and wide- reaching in application, targeting large samples or accessing hard-to-reach groups. It is thought that the worldwide „digital divide" caused by rapid and uneven growth of information and communication technologies is less pronounced for mobile phones than for other technologies such as the Internet (Campbell, 2001) probably due to the lower level of skills required to use mobile phones compared with Internet and email, thus making phones more widely accessible, although they may not be suitable for all. Further, mobile phones carry risks of security and privacy breach in the transmission of health information that need to be addressed before mobile technology can become commonplace in health communication.

This overview of current developments, although not exhaustive, has shown that research in this area is promising. We will undoubtedly see a dramatic increase in the application of mobile technologies in healthcare over coming years, with many unevaluated interventions already currently in place. At present, mobile phone intervention is an emerging but rapidly advancing field and research evidence, although positive, is limited, particularly in the UK. More quality research is needed to provide evidence of effectiveness for the impact of mobile phones on health knowledge, health outcomes and healthcare delivery.

Key Points

1. Developments in information and communications technology have seen a rise in applications for mobile phones in healthcare

2. Mobile phone interventions have shown promising results for health promotion and chronic disease management

3. Rapid advances in technology mean that mobile phones will increasingly be used in patient care to improve nurse-patient communications 


\section{References}

Anhøj, J., \& Møldrup, C. (2004). Feasibility of collecting diary data from asthma patients through mobile phones and SMS (short message service): response rate analysis and focus group evaluation from a pilot study. Journal of Medical Internet Research, 6(4), e42.

Bielli, E., Carminati, F., Capra, S. L., Lina, M., Brunelli, C., \& Tamburini, M. (2004). A Wireless Health Outcomes Monitoring System (WHOMS): development and field testing with cancer patients using mobile phones. BMC Medical Information and Decision Making, 4(7).

Bramley, D., Riddell, T., Whittaker, R., Corbett, T., Lin, R., Wills, M., et al. (2005). Smoking cessation using mobile phone text messaging is as effective in Maori as non-Maori. New Zealand Medical Journal, 118(1216), U1494.

Campbell, D. (2001). Can the digital divide be contained? . International Labour Review, 140(2).

Carroll, A., Marrero, D., \& Downs, S. (2007). The HealthPia GlucoPack Diabetes phone: a usability study. Diabetes Technol Ther, 9(2), 158-164.

Collins, R., Kashdan, T., \& Gollnisch, G. (2003). The feasibility of using cellular phones to collect ecological momentary assessment data: application to alcohol consumption. Exp Clin Psychopharmacol, 11(1), 73-78.

Consolvo, S., Everitt, K., Smith, I., \& Landay, J. (April 22-27 2006). Design Requirements for Technologies that Encourage Physical Activity. Paper presented at the CHI: Designing for Tangible Interactions, Montréal, Québec, Canada.

Farmer, A., Gibson, O., Hayton, P., Bryden, K., Dudley, C., Neil, A., et al. (2005). A realtime, mobile phone-based telemedicine system to support young adults with type 1 diabetes. Inform Prim Care, 13(3), 171-177.

Freedman, M., Lester, K., McNamara, C., Milby, J., \& Schumacher, J. (2006). Cell phones for ecological momentary assessment with cocaine-addicted homeless patients in treatment. Journal of Substance Abuse \& Treatment, 30(2), 105-111.

Gammon, D., Arsand, E., Walseth, O., Andersson, N., Jenssen, M., \& Taylor, T. (2005). Parent-child interaction using a mobile and wireless system for blood glucose monitoring. Journal of Medical Internet Research, 7(5), e57.

Giménez-Pérez, G., Gallach, M., Acera, E., Prieto, A., Carro, O., Ortega, E., et al. (2002). Evaluation of accessibility and use of new communication technologies in patients with type 1 diabetes mellitus. Journal of Medical Internet Research, 4(3), E16.

Hurling, R., Catt, M., Boni, M., Fairley, B., Hurst, T., Murray, P., et al. (2007). Using 
internet and mobile phone technology to deliver an automated physical activity program: randomized controlled trial. journal of Medical Internet Research, 9(2), e7.

Kikunaga, S., Tin, T., Ishibashi, G., Wang, D., \& Kira, S. (2007). The application of a handheld personal digital assistant with camera and mobile phone card (Wellnavi) to the general population in a dietary survey. J Nutr Sci Vitaminol (Tokyo), 53(2), 109-116.

Kikunaga, S., Tin, T., Ishibashi, G., Wang, D., \& Kira, S. (2007). The application of a handheld personal digital assistant with camera and mobile phone card (Wellnavi) to the general population in a dietary survey. J Nutr Sci Vitaminol (Tokyo), 53(2), 109-116.

Kim, H. (2007). A randomized controlled trial of a nurse short-message service by cellular phone for people with diabetes. International Journal of Nursing Studies, 44(5), 687-692.

Kim, H., \& Jeong, H. (2007). A nurse short message service by cellular phone in type2 diabetic patients for six months. Journal of Clinical Nursing, 16(6), 1082- 1087.

Kubota, A., Fujita, M., \& Hatano, Y. (2004). [Development and effects of a health promotion program utilizing the mail function of mobile phones] [Article in Japanese]. Nippon Koshu Eisei Zasshi, 51(10), 862-873.

Lazev, A., Vidrine, D., Arduino, R., \& Gritz, E. (2004). Increasing access to smoking cessation treatment in a low-income, HIV-positive population: the feasibility of using cellular telephones. Nicotine \& Tobacco Research, 6(2), 281- 286.

Miskelly, F. (2005). Electronic tracking of patients with dementia and wandering using mobile phone technology. Age \& Ageing, 34(5), 497-499.

Obermayer, J., Riley, W., Asif, O., \& Jean-Mary, J. (2004). College smoking- cessation using cell phone text messaging. Journal of American College Health, 53(2), 71-78.

Rodgers, A., Corbett, T., Bramley, D., Riddell, T., Wills, M., Lin, R., et al. (2005). Do u smoke after txt? Results of a randomised trial of smoking cessation using mobile phone text messaging. Tobacco Control, 14(4), 255-261.

Vidrine, D., Arduino, R., Lazev, A., \& Gritz, E. (2006a). A randomized trial of a proactive cellular telephone intervention for smokers living with HIV/AIDS. AIDS, 20(2), 253-260.

Vidrine, D., Arduino, R., \& Gritz, E. (2006b). Impact of a cell phone intervention on mediating mechanisms of smoking cessation in individuals living with HIV/AIDS. Nicotine \& Tobacco Research, 8(Suppl 1), S103-108. 
Wang, D., Kogashiwa, M., \& Kira, S. (2006). Development of a new instrument for evaluating individuals' dietary intakes. Journal of the Americal Diet Association, 106(10), 1588-1593.

Wang, D., Kogashiwa, M., Ohta, S., \& Kira, S. (2002). Validity and reliability of a dietary assessment method: the application of a digital camera with a mobile phone card attachment. J Nutr Sci Vitaminol (Tokyo), 48(6), 498-504.

Wilkins, C., Casswell, S., Barnes, H., \& Pledger, M. (2003). A pilot study of a computer-assisted cell-phone interview (CACI) methodology to survey respondents in households without telephones about alcohol use. Drug \& Alcohol Reviews, 22(2), 221-225. 